\title{
Mathematical modeling of heat-trapping properties of a developed radiator surface in mechanical engineering
}

\author{
Nikolay Chernov ${ }^{1}$, Alexander Palii ${ }^{2}$, Larisa Tolmacheva ${ }^{2}$, Konstantin Tomilin $^{2,}$, and \\ Mikhail Kritskiy ${ }^{2}$ \\ ${ }^{1}$ Southern Federal University, Nekrasovski all., 44, 344922, Taganrog, Russia \\ ${ }^{2}$ Politechnicheskiy Institute (branch) of DSTU in Taganrog, Petrovskaya str., 109a, 347900, Russia
}

\begin{abstract}
In this work the research of heat-removing properties of areas of extended surfaces, for example, of finned and needle radiators for assessment of efficiency of distribution of a thermal field and heat extraction is described. Also the description of the carried-out computational modeling is provided in the Fluent processor of universal program system of the final and element analysis Ansys. The relevance of the selected subject is confirmed by the fact that one of the most important and difficult tasks arising when developing the electronic equipment is a withdrawal of heat generated by it. At a current steady trend in reduction of dimensions of electronic devices this problem does not disappear, and opposite, becomes more and more sharp, and that is stronger, than device high power less its physical volume, and not only the efficiency of heat extraction, but also dimensions and, of course, reliability of operation of electronic devices depends on constructions of heat-removing elements. In work the conclusion is drawn that for a solution of a complex problem of assessment of efficiency of the heat sink for the purpose of decrease in temperature of heatterminated element, it is necessary to use electrothermal analogy.
\end{abstract}

\section{Introduction}

Ensuring regulatory thermal regimes electronic means is that the temperature field in the system in which all the elements are working in an environment that meets the technical requirements, is one of the key tasks in the design. The main way to ensure the required thermal regime is to create a thermal dynamic equilibrium, that is, to create conditions under which the amount of heat dissipated in the environment is equal to the amount of heat released in the device. The main losses in power transistors and modules are created directly in the crystal. The heat generated by the semiconductor crystal is diverted through the cell body to the heat sink and then to the environment $[1,2]$. The heat from the source is removed primarily through the materials that are in direct contact with it, i.e. the element body and the heat sink. Since the heat sink has finite dimensions, its temperature is always higher $[3,4]$.

\footnotetext{
*Correcting author: kostyanich2000@mail.ru
} 


\section{Problem statement}

Let there be heat power source $W=\frac{d Q}{d t}$ generates thermal field whose flow $\bar{h}$ per unit time, per unit surface characterizes the heat load of the body surface source [5].

As applied, for example, to electronic equipment, the source of thermal power is a heatloaded element. In the case when the thermal conductivity and convection of a liquid or gas is not enough to normalize its thermal regime, heat sinks (radiators) are used - heat-removing structures made of metal with a large coefficient of thermal conductivity (copper, aluminum) since the coefficient of thermal conductivity in metals is tens of thousands of times higher than that of air [6].

To solve the complex problem of evaluating the efficiency of the surface areas of the heat sink in order to reduce the temperature of the heat-loaded element, it is necessary to study the surface areas from the point of view of the propagation of the thermal field by applying the electrothermal analogy [7].

\section{Theoretical intelligence}

The lines of force of the heat field are called the heat flow $\bar{h}$. The heat flow from a point source at a distance of radius $\mathrm{R}$ is equal to:

$$
\bar{h}=\frac{W}{4 \pi \lambda R^{2}},
$$

where $\lambda$ - coefficient of thermal conductivity of the medium, $W$ - power of the heat source.

The heat flow propagates perpendicular to the equitemperature lines and is related to the temperature by the following relation:

$$
\bar{h}=-\operatorname{grad} T
$$

The surface temperature of a ball with radius $\mathrm{R}$ with a heat source in its center will be equal to:

$$
T=\frac{W}{4 \pi \lambda R},
$$

The heat capacity of this ball will be equal to:

$$
C=4 \pi \lambda R
$$

This ratio means that the heat capacity is determined by the ratio of the power of the heat source to the temperature change.

$$
C=\frac{W}{T} .
$$

If now in the heat field of the source there is a heat sink (a body with a high coefficient of thermal conductivity) with a developed surface (needle, pin, plate, etc.), then the field will be distorted.

To solve this problem and the possibility of applying the electrothermal analogy for the given parameters (1) - (5), it is necessary to choose similar electrostatic ones, since the equations for the propagation of thermal and electromagnetic fields are similar and have 
similar solutions[8-9]. The symbols used may, be different, but the mathematical form of the equations is the same.

An electrostatic field has characterized by lines of force or intensity:

$$
\bar{E}=\frac{\bar{F}}{q},
$$

where $\bar{F}$ - the force acting from the field on a stationary charge $q$.

The intensity of the electrostatic field of the ball or point charge will be equal to:

$$
\bar{E}=\frac{q}{4 \pi \varepsilon_{0} r^{2}} .
$$

which is similar (1).

The lines of force or the strength of the electrostatic field at any point are perpendicular to the equipotential surfaces and directed in the direction of decreasing potential. The relationship between the strength and the potential of the electrostatic field seen from (8):

$$
\bar{E}=-\operatorname{grad} \varphi \text {. }
$$

which is similar (2).

The potential of the field of the ball of radius $R$ with a charge $q$, evenly distributed over its surface is equal to:

$$
\varphi=\frac{q}{4 \pi \varepsilon_{0} \varepsilon R}
$$

which is similar (3).

The electrical capacity of the ball will be equal to:

$$
C=4 \pi \varepsilon_{0} \varepsilon R
$$

which is similar (4).

The electrical capacity of a conductor has called the value $C$, numerically equal to the charge that changes the potential of the conductor by one unit:

$$
C=\frac{q}{\varphi} .
$$

which is similar (5).

The obtained electrothermal analogies may be summarized in Table 1.

The method of electrostatic analogy in most cases does not give an analytical expression of solutions; the only general method of solutions is numerical. However, in a number of specific cases, it is possible to find an analytical solution if we consider the theory of functions of a complex variable [10-11]. 
Table 1. The same parameters.

\begin{tabular}{|c|c|}
\hline Electrostatic field & Temperature field \\
\hline$\varphi$ - potential & $T$ - temperature \\
\hline$Q$ - charge & $W$ - power output \\
\hline $\bar{E}$ - tension & $\bar{h}$ - flow \\
\hline$\varepsilon$ - conductivity coefficient & $\lambda$ - coefficient of thermal conductivity \\
\hline$C=Q / \varphi$ - electrical capacity & $C=W / T$ - heat capacity by analogy \\
\hline$C=4 \pi \varepsilon o \varepsilon R$ - electrical capacity of the ball & $C=4 \pi \lambda R$ - heat capacity of the ball \\
\hline
\end{tabular}

The theory of a complex variable is based on the presence of the imaginary part of a number. Such a complex number can be represented as two vectors whose lengths correspond to the values of the real and imaginary parts, and the direction or angle between the vectors can be any, since one of the parts of the number does not exist. The most convenient, and in our case also, should be considered an angle of ninety degrees, which has already become a classic, and other angles are no longer used. The property of the perpendicularity of the two parts of the function of a complex variable automatically leads to the solution of the Laplace equation [12-13].

\section{Proposed model}

This theoretical study, in addition to confirming the electrothermal analogies, can be confirmed by conducting a computational experiment in the Ansys Fluent program. The simulation was performed in the processor of the Fluent-universal software system of finite element analysis Ansys [14-16]. In the SolidWorks program, three-dimensional models of the heat-loaded element and the heat-removing body under study were created. The Ansys Workbench program developed project files containing all the necessary complexes for studying the propagation of temperature fields in the body-flow system. The designed geometry of the workspace was later transformed into a grid structure, where the calculated grid was designed using the Ansys Meshing software component. Next, the thermal radiation of the source was simulated at a power of $5 \mathrm{~W}$. Then, based on the constructed models, a grid with the number of elements 3270991 was developed. Then the stage of static modeling of heat radiation by the source in the region without the influence of external air flow was carried out at an external temperature (including the initial temperature of the radiator) of $295 \mathrm{~K}$ with a heat transfer coefficient (copper/air) of $11.3 \mathrm{~W} / \mathrm{m}^{\wedge} 2 * \mathrm{~K}$ [17-19].

The following temperature distributions were obtained, as shown in Figure I.I. 


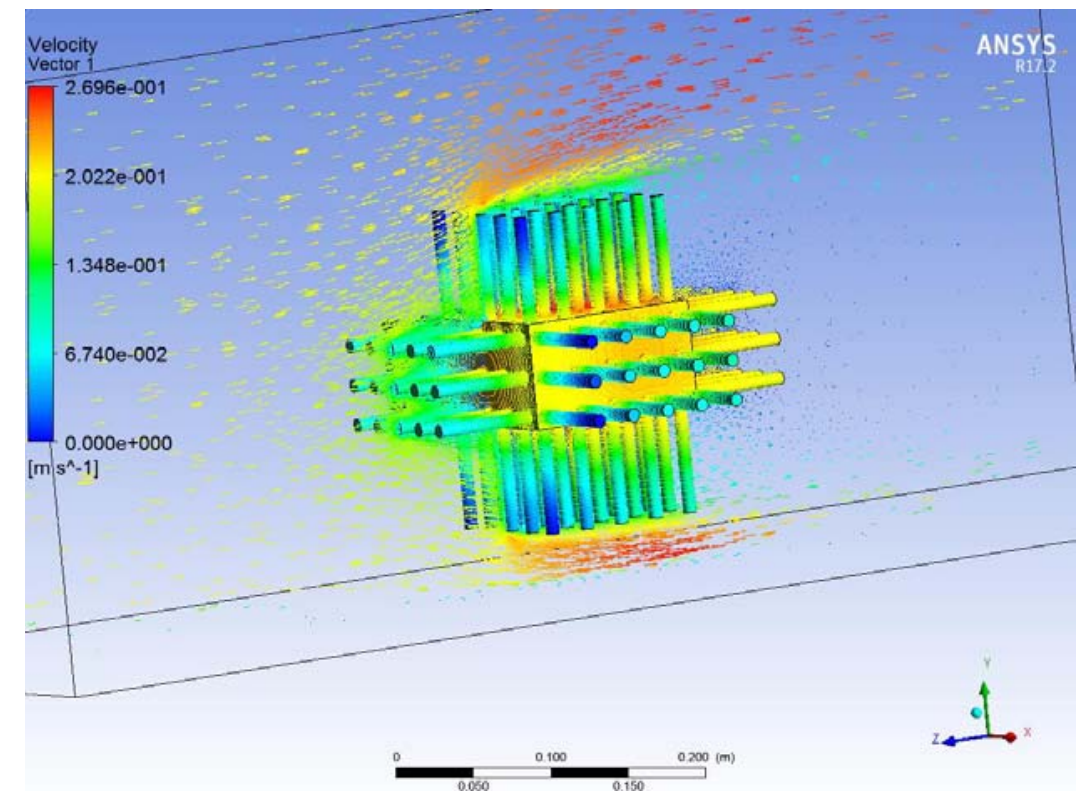

Fig. 1. Heat field from the pin heat sink.

Figure 1 shows that the farther away the heat sink area (the tops of the pins) is, the less efficient this area becomes.

\section{Conclusions}

Thus, the article describes the study of the heat-dissipation properties of the areas of developed radiator surfaces to assess the efficiency of the propagation of the thermal field and heat removal. The article also describes the numerical simulation performed in the Fluent processor, a universal software system for finite element analysis (Ansys). The relevance of the chosen topic is confirmed by the fact that one of the most important and complex tasks that arise in the development of electronic equipment is the removal of heat generated by it.

To solve the complex problem of evaluating the efficiency of the surface areas of the heat sink in order to reduce the temperature of the heat-loaded element, it is necessary to study the surface areas from the point of view of the propagation of the thermal field by applying the electrothermal analogy.

The method of electrothermal analogy in most cases does not give an analytical expression of solutions, the only general method of solutions is numerical. But in a number of specific cases, it is possible to find an analytical solution if we consider the theory of functions of a complex variable.

The conducted computational experiment confirmed the theoretical assumption that the presence of pins, plates and fins on the surface of the heat sink, despite a significant increase in the surface area, does not lead to an increase in the effective area of heat dissipation.

\section{References}

1. V.I.Konovalov, A.N.Pakhomov, N.S.Gatapova, A.N. Koliukh, Methods for solving heat and mass transfer problems. Thermal conductivity and diffusion in a stationary medium (Tambov, 2012) 
2. B. Turan, H.F. Oztop, Heat engineering 16(1), 139-149 (2012)

3. Yu.I. Dimitrienko, Solid Mechanics and its Applications 224, 269-307 (2016)

4. A.V. Palii, E.T. Zamkov, News SFU. Technical sciences 1(138), 197-202 (2013)

5. A.V. Probudlsky, N.N. Chernov, A.V. Palii, Technical sciences 2(196), 155-163 (2018)

6. B.T. Kuzhiveli, IOP Conference Series: Materials Science and Engineering 278(1), 012136 (2017)

7. B. Nita, P. Nolan, A. Vaidya, Computational and Applied Mathematics 36(4), 17331746 (2017)

8. X. Wu, F. Chen, H. Liu, Applied Mathematics and Mechanics 38(12), 1679-1696 (2017)

9. V.I. Zvegintsev, S.O. Morozov, D.G. Nalivaychenko, AIP Conference Proceedings 2027, 030012 (2018)

10. V.N. Timofeev, Journal of Physics: Conference Series 1141(1), 012095 (2018)

11. I.V. Egorov, I.V. Ezhov, A.V. Novikov et al., 53rd AIAA Aerospace Sciences Meeting, 189-197 (2015)

12. P. De Palma, M. D.de Tullio, G. Pascazio et al., 17th AIAA Computational Fluid Dynamics Conference, 37-42 (2015)

13. S. Chen, Y. Hu, Q. Sun, AIP Conference Proceedings 1501(1), 1515-1521 (2012)

14. S.P. Malyukov, A.V. Sayenko, S.S. Zinovev, Proceedings - International Russian Automation Conference, RusAutoCon 2020 9208191, 368-373 (2020)

15. S. Pradhan, V. Kumaran, 14th International Energy Conversion Engineering Conference, 32-42 (2016)

16. S.A. Isaev, Y.M. Lipnitskii, A.N. Mikhalev et al., Journal of Engineering Physics and Thermophysics 84(4), 827-839 (2011)

17. W. Wang, J. Wang, X.P. Yang, Y.Y. Ding, Journal of Thermal Science and Engineering Applications 11(2), 021015 (2019)

18. S. Pradhan, 8th AIAA Theoretical Fluid Mechanics Conference, 214-220 (2017) 